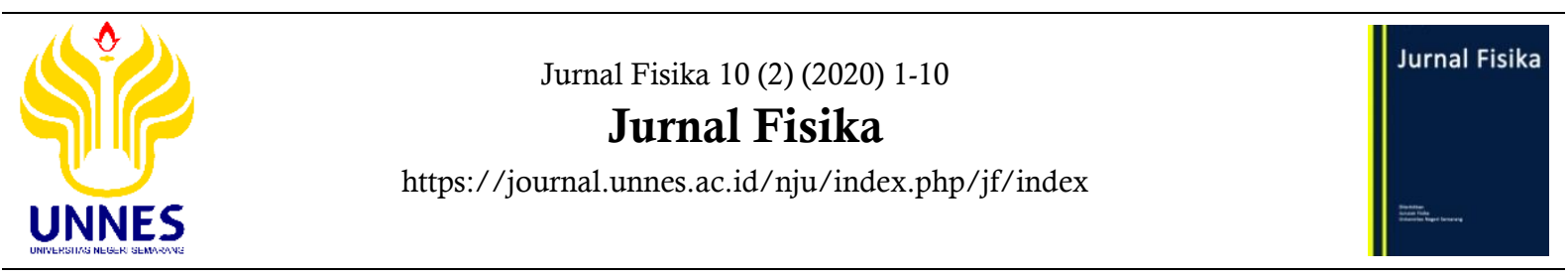

\title{
The Analytical Solutions of the Schrodinger Equation for a Single Electron in the Nikiforov-Uvarov Framework
}

\section{Yacobus Yulianto ${ }^{\bowtie}$ and Zaki Su'ud}

Advanced Nuclear Laboratory, Physics Department, FMIPA, Institut Teknologi Bandung Jl. Ganesha 10, Bandung 40132, Indonesia

\footnotetext{
Article Info

Article History

\section{ABSTRACT}

Received:

02 Juli 2020

Accepted:

23 Desember 2020

Published:

25 Desember 2020

It is very interesting to study about the electron related to the Schrodinger equation. To explore the characteristics of each electron in the system, quantum mechanics with the mathematical method, can be a useful tool to explain this problem. Based on this idea, this study is intended to show an alternative method to derive the wave function of a single electron as solutions of the Schrodinger equation with Coulomb potential. The Nikiforov-Uvarov method has been chosen to be utilized in this study since this method can solve the Schrodinger equation with several well-known potentials in the non-relativistic mechanics of quantum. The obtained results of this study have succeeded to derive the single-electron wave function from the Schrodinger equation. This single-electron wave function is similar to the single-electron wave function written in quantum physics textbooks. These results prove that the Nikiforov-Uvarov method provides an

Keywords: alternative procedure to solve the Schrodinger equation.

a single electron,

Nikiforov-Uvarov

method, Schrodinger

equation, quantum
} 


\section{INTRODUCTION}

In non-relativistic quantum mechanics, all important information about the physical properties of a system can be extracted from the wave function. It is very important to understand about the wave function of each electron in the system which can provide many important information about the system. This wave function is the solution of the Schrodinger equation and has a relation to the eigenenergy of the system. The analytical solutions of the Schrodinger equation for several different potentials have been obtained and widely used in advanced fields of molecular, atomic, nuclear, and sub-nuclear physics. Up to now, many methods and approaches have been employed to solve the Schrodinger equation analytically.

One of these methods is the Nikiforov-Uvarov (NU) method (Nikiforov \& Uvarov, 1988). This method has been successful to accomplish different second-order differential equations in nonrelativistic quantum mechanics (Ikot \& Akpan, 2012; Pahlavani \& Alavi, 2012; Bayrak \& Aciksoz, 2015; Okon et al., 2017). This method has recently been used to find reliable Schrodinger equation solutions for many well-known potentials in the non-relativistic mechanics of quantum, such as harmonic oscillator (Antia et al., 2018), Kratzer (Edet et al., 2019), Hulthen (Agboola, 2011), HulthenManning-Morse (Meyur \& Debnath, 2009), and pseudoharmonic (Sever et al., 2007). In this study, we have utilized the NU method to extract the single-electron wave function. It is intended that the result of this study can enrich the alternative way to solve the Schrodinger equation in explaining the single-electron wave function as lectured in quantum mechanics textbooks.

The main purposes of this study are to solve the Schrodinger equation analytically by using the NU method and to obtain the wave function and the energy eigenvalue for a single electron. This paper is organized as follows. In Section 2, it is explained the Schrodinger equation for a single electron. Then, the NU method is briefly explained in Section 3. The analytical solutions as the obtained results of this study are displayed in Section 4. Finally, the summary of this study is represented in Section 5.

\section{THE SCHRODINGER EQUATION FOR A SINGLE ELECTRON}

As a fundamental parameter to study the atomic structure and to build the wave function of the atom, the single-electron wave function is derived from the Schrodinger equation. One can write the Schrodinger equation for a central potential as

$$
-\frac{\hbar^{2}}{2 \mu} \nabla^{2} \Psi_{n_{p}, l, m}(r, \theta, \phi)+V(r) \Psi_{n_{p}, l, m}(r, \theta, \phi)=E \Psi_{n_{p}, l, m}(r, \theta, \phi)
$$

where $\hbar$ is the reduced Planck constant, $\mu$ is the reduced mass of the nucleus, E is the energy eigenvalue, and $\Psi_{\mathrm{n}_{\mathrm{p}}, \mathrm{l}, \mathrm{m}}(\mathrm{r}, \theta, \varphi)$ is the wave function in spherical coordinate. In atomic unit, the Coulomb potential $\mathrm{V}(\mathrm{r})$ of a single electron is approached as

$$
V(r)=-\frac{Z^{2}}{r}-\frac{\hbar^{2} l(l+1)}{2 \mu r^{2}}
$$

where $\mathrm{Z}$ is the atomic number, e is the charge of the electron, $\mathrm{r}$ is the distance between the electron and the nucleus, and $\mathrm{l}$ is the momentum angular quantum number. By introducing the total wave function, $\Psi_{\mathrm{n}_{\mathrm{p}}, \mathrm{l}, \mathrm{m}}(\mathrm{r}, \theta, \varphi)=\mathrm{R}(\mathrm{r}) \Theta(\theta) \Phi(\phi)$, which is normalized by $\int \Psi^{*} \Psi \mathrm{d}^{3} \mathrm{r}=1$, and in spherical coordinate, one can write Eq. (1) as 


$$
\begin{aligned}
-\frac{\hbar^{2}}{2 \mu}\left[\frac{1}{\mathrm{r}^{2}} \frac{\partial}{\partial \mathrm{r}}\left(\mathrm{r}^{2} \frac{\partial}{\partial \mathrm{r}}\right)+\frac{1}{\mathrm{r}^{2}}\left(\frac{1}{\sin \theta}\right.\right. & \left.\left.\frac{\partial}{\partial \theta}\left[\sin \theta \frac{\partial}{\partial \theta}\right]\right)+\frac{1}{\mathrm{r}^{2}} \frac{1}{\sin ^{2} \theta} \frac{\partial^{2}}{\partial \phi^{2}}\right] \mathrm{R}(\mathrm{r}) \Theta(\theta) \Phi(\phi) \\
+ & {\left[-\frac{\mathrm{Ze}}{\mathrm{r}}-\frac{\hbar^{2} \mathrm{l}(\mathrm{l}+1)}{2 \mu \mathrm{r}^{2}}\right] \mathrm{R}(\mathrm{r}) \Theta(\theta) \Phi(\phi)=\operatorname{ER}(\mathrm{r}) \Theta(\theta) \Phi(\phi) }
\end{aligned}
$$

where $\mathrm{R}(\mathrm{r})$ is the radial function, $\Theta(\theta)$ is the polar function, and $\Phi(\phi)$ is the azimuthal function. By using the separation variable, it can be obtained three equations as follows

$$
\begin{gathered}
\frac{\mathrm{d}^{2} \mathrm{R}(\mathrm{r})}{\mathrm{dr} \mathrm{r}^{2}}+\frac{2}{\mathrm{r}} \frac{\mathrm{dR}(\mathrm{r})}{\mathrm{dr}}+\frac{2 \mu}{\hbar^{2}}\left[\mathrm{E}+\frac{\mathrm{Ze}^{2}}{\mathrm{r}}-\frac{\hbar^{2} \mathrm{l}(\mathrm{l}+1)}{2 \mu \mathrm{r}^{2}}\right] \mathrm{R}(\mathrm{r})=0 \\
\frac{\mathrm{d}^{2} \Theta(\theta)}{\mathrm{d} \theta^{2}}+\frac{\cos \theta}{\sin \theta} \frac{\mathrm{d} \Theta(\theta)}{\mathrm{d} \theta}+\left\{\mathrm{l}(\mathrm{l}+1)-\frac{\mathrm{m}^{2}}{\sin ^{2} \theta}\right\} \Theta(\theta)=0 \\
-\frac{\hbar^{2}}{2 \mu}\left[\frac{1}{\mathrm{r}^{2}} \frac{1}{\sin ^{2} \theta} \frac{\partial^{2}}{\partial \phi^{2}}\right] \Phi(\phi)-\frac{2 \mu}{\hbar^{2}} \frac{\mathrm{m}^{2}}{\mathrm{r}^{2} \sin ^{2} \theta} \Phi(\phi)=0
\end{gathered}
$$

where $\mathrm{m}$ is the magnetic quantum number respectively (Eisberg, 1974; Griffith, 1995; Zettili, 2009). To solve these three equation, it is presented an alternative procedure by using the NU method.

\section{THE NIKIFOROV-UVAROV (NU) METHOD}

The NU method is constructed to accomplish the hypergeometric type second-order differential equations through the special orthogonal functions. This method can be performed to solve the following second-order differential equations with a suitable coordinate transformation where

$$
\frac{\mathrm{d}^{2} \psi_{\mathrm{n}}(\mathrm{s})}{\mathrm{d} \mathrm{s}^{2}}+\frac{\tilde{\tau}(\mathrm{s})}{\sigma(\mathrm{s})} \frac{\mathrm{d} \psi_{\mathrm{n}}(\mathrm{s})}{\mathrm{d} s}+\frac{\tilde{\sigma}(\mathrm{s})}{\sigma^{2}(\mathrm{~s})} \psi_{\mathrm{n}}(\mathrm{s})=0
$$

where $\sigma(s)$ and $\widetilde{\sigma}(\mathrm{s})$ are the mostly second-order polynomials. The parameter $\tilde{\tau}(\mathrm{s})$ is a first-order polynomial. Separating the variables and applying the following assumption, one can formulate the function $\psi_{\mathrm{n}}(\mathrm{s})$ as

$$
\psi_{\mathrm{n}}(\mathrm{s})=\phi_{\mathrm{n}}(\mathrm{s}) \mathrm{y}_{\mathrm{n}}(\mathrm{s})
$$

where the function $\phi(s)$ can be determined by using

$$
\frac{\mathrm{d} \phi(\mathrm{s})}{\mathrm{ds}} \frac{1}{\phi(\mathrm{s})}=\frac{\pi(\mathrm{s})}{\sigma(\mathrm{s})}
$$

The function $\mathrm{y}_{\mathrm{n}}(\mathrm{s})$ in Eq. (8) is the hypergeometric form function in which the Rodrigues relation provides polynomial solutions as

$$
\mathrm{y}_{\mathrm{n}}(\mathrm{s})=\frac{\mathrm{B}_{\mathrm{n}}}{\rho(\mathrm{s})} \frac{\mathrm{d}^{\mathrm{n}}}{\mathrm{ds^{ \textrm {n } }}}\left\{\sigma^{\mathrm{n}}(\mathrm{s}) \rho(\mathrm{s})\right\}
$$

where $B_{n}$ is a normalization constant. The function $\rho(s)$ can be analyzed by using 


$$
\frac{\mathrm{d} \rho(\mathrm{s})}{\mathrm{ds}} \frac{1}{\rho(\mathrm{s})}=\frac{\tau(\mathrm{s})-\sigma^{\prime}(\mathrm{s})}{\sigma(\mathrm{s})}
$$

Next, the expressions of

$$
\lambda_{\mathrm{n}}=-\mathrm{n} \tau^{\prime}(\mathrm{s})-\frac{\mathrm{n}(\mathrm{n}-1)}{2} \sigma^{\prime \prime}(\mathrm{s})
$$

and

$$
\lambda=\mathrm{k}+\pi^{\prime}(\mathrm{s})
$$

are equalized to yield the energy eigenvalue. The superscript ' and " are the first and the second derivatives of the functions respectively. The parameter $\tau(\mathrm{s})$ can be gained by using

$$
\tau(s)=\tilde{\tau}+2 \pi(s)
$$

The $\pi(\mathrm{s})$ parameter is yielded by solving the resulting quadratic equation as follows

$$
\pi(s)=\frac{\sigma^{\prime}(s)-\tilde{\tau}(s)}{2} \pm \sqrt{\left\{\frac{\sigma^{\prime}(s)-\tilde{\tau}(s)}{2}\right\}^{2}-\widetilde{\sigma}(s)+k \sigma(s)}
$$

The determination of $\mathrm{k}$ is the crucial point in the calculation to solve Eq. (15). The $\mathrm{k}$ value can be determined by setting the discriminant of the square root to zero (Nikiforov \& Uvarov, 1988).

\section{ANALYTICAL SOLUTIONS}

\section{The Solution to Radial Equation}

In this section, the NU method was employed to achieve the solution to radial equation. To solve Eq. (4) one should consider the centrifugal approximation, i.e.

$$
\frac{\mathrm{d}^{2} \mathrm{R}(\mathrm{r})}{\mathrm{dr}^{2}}+\frac{2}{\mathrm{r}} \frac{\mathrm{dR}(\mathrm{r})}{\mathrm{dr}}+\frac{1}{\mathrm{r}^{2}}\left\{\frac{2 \mu \mathrm{E}}{\hbar^{2}} \mathrm{r}^{2}+\frac{2 \mu \mathrm{Ze} \mathrm{e}^{2}}{\hbar^{2}} \mathrm{r}-\mathrm{l}(\mathrm{l}+1)\right\} \mathrm{R}(\mathrm{r})=0
$$

By using the transformation, Eq. (16) becomes

$$
\frac{\mathrm{d}^{2} \psi(\mathrm{s})}{\mathrm{ds}^{2}}+\frac{2}{\mathrm{~s}} \frac{\mathrm{d} \psi(\mathrm{s})}{\mathrm{ds}}+\frac{\left[-\mathrm{A}^{2} \mathrm{~s}^{2}+\mathrm{Bs}-\mathrm{C}\right]}{\mathrm{s}^{2}} \psi(\mathrm{s})=0
$$

where

$$
-\mathrm{A}^{2}=\frac{2 \mu \mathrm{E}}{\hbar^{2}}, \quad \mathrm{~B}=\frac{2 \mu \mathrm{Ze}^{2}}{\hbar^{2}}, \quad \mathrm{C}=\mathrm{l}(\mathrm{l}+1)
$$

By comparing Eq. (7) and Eq. (17), one can determine the following

$$
\tilde{\tau}(s)=2, \sigma(s)=s, \quad \widetilde{\sigma}(s)=-A^{2} s^{2}+\beta s-C
$$

Substituting these expressions in Eq. (19) into Eq. (15), one has 


$$
\pi(s)=-\frac{1}{2} \pm \frac{1}{2} \sqrt{4 A^{2} s^{2}+(4 k-4 B) s+1+4 C}
$$

The constant parameter $\mathrm{k}$ can be determined by applying the zero discriminant for the rootpart, i.e.

$$
\mathrm{k}=\mathrm{B} \pm \mathrm{A} \sqrt{1+4 \mathrm{C}}
$$

Thus, there are four possible solutions for Eq. (20), i.e.

$$
\pi(s)=\left\{\begin{array}{l}
-\frac{1}{2} \pm \frac{1}{2}\{2 A s+\sqrt{1+4 C}\} \text { for } k=B+A \sqrt{1+4 C} \\
-\frac{1}{2} \pm \frac{1}{2}\{2 A s-\sqrt{1+4 C}\} \text { for } k=B-A \sqrt{1+4 C}
\end{array}\right.
$$

Since Eq. (14) should have a negative derivative physically (for the bound-state case), the valid solution is

$$
\pi(s)=-\frac{1}{2}-\frac{1}{2}\{2 \mathrm{As}-\sqrt{1+4 \mathrm{C}}\}
$$

for $\mathrm{k}=\mathrm{B}-\mathrm{A} \sqrt{1+4 \mathrm{C}}$ and it yields

$$
\tau(s)=1+\sqrt{1+4 C}-2 A s
$$

Then, by using Eq. (12) and (13), it can be defined that

$$
2 \mathrm{nA}=\mathrm{B}-\mathrm{A} \sqrt{1+4 \mathrm{C}}-\mathrm{A}
$$

for $\mathrm{n}=1,2,3, \ldots$ Then, assisted by Eq. (18), Eq. (25) can produce the energy eigenvalue as

$$
E_{n_{p}}=-\frac{1}{2} \mu c^{2} \frac{Z^{2} \alpha^{2}}{n_{p}^{2}}
$$

where $n_{p}=n+1+l$ is well-known as the principal quantum number. It should be noted that $n$ is the non-physics integer number. Next, to obtain the wave function, $\pi(s)$ from Eq. (23) and $\sigma(s)$ from Eq. (19) were substituted into Eq. (9). By solving the first-order differential equation, one can obtain

$$
\phi(s)=s^{1} e^{-s A}
$$

For the weight function $\rho(s)$, it can be determined by using

$$
\rho(s)=s^{2 l+1} e^{-2 A s}
$$

Substituting Eq. (28) into the Rodrigues relation in Eq. (10), one has

$$
y_{n}(s)=\frac{1}{n !} e^{2 A s} s^{-(2 l+1)} \frac{d^{n}}{d s^{n}}\left\{s^{n+2 l+1} e^{-2 A s}\right\}
$$

Therefore, based on Eq. (8), the wave function can be formulated as 


$$
\psi_{n, 1}(s)=N_{n, l}\left\{s^{l} e^{-s A}\right\}\left\{\frac{1}{n !} e^{2 A s} s^{-(21+1)} \frac{d^{n}}{d s^{n}}\left\{s^{n+21+1} e^{-2 A s}\right\}\right\}
$$

Therefore, by using Eq. (18), $\mathrm{n}_{\mathrm{p}}=\mathrm{n}+1+\mathrm{l}$, and $\mathrm{r}^{-1}=\mathrm{z}\left(\mathrm{n}_{\mathrm{p}}^{2} \mathrm{a}_{0}\right)^{-1}$ (Gasiorowicz, 2000) the combined wave function can be formulated as

$$
\Psi_{n, l}(r)=N_{n, 1}\left\{s^{1} e^{-\frac{Z r}{(n+1+1) a_{0}}}\right\}\left\{\frac{1}{n !} e^{\frac{2 Z r}{(n+l+1) a_{0}}} r^{-(2 \cdot l+1)} \frac{d^{n}}{d^{n}}\left\{r^{n+2 l+1} e^{-\frac{2 Z r}{(n+1+1) a_{0}}}\right\}\right\}
$$

where $a_{0}$ is the Bohr radius and $\mathrm{N}_{n, 1}$ is the normalization constant. Because $\mathrm{n}$ is not a physical property, it should be taken good care to obtain the radial wave function. It is needed the relation between the radial wave function $\mathrm{R}(\mathrm{r})$ and the function $\psi(\mathrm{r})$. The equalization between those function can be formulated as

$$
\mathrm{R}_{\mathrm{n}_{\mathrm{p}}, 1}(\mathrm{r})=\Psi_{\mathrm{n}, 1}(\mathrm{r})=\Psi_{\mathrm{n}_{\mathrm{p}}-1-1,1}(\mathrm{r})
$$

\section{The Solution to Angular Equation}

To obtain the solutions to the angular equation, it is commenced by using Eq. (5). To meet the requirement of Eq. (7), by using the transformation variable, the form of Eq. (5) is transformed to be

$$
\frac{d^{2} \psi(s)}{d s^{2}}-\frac{2 s}{\left(1-s^{2}\right)} \frac{d \psi(s)}{d s}+\frac{\left\{l(l+1)\left(1-s^{2}\right)-m^{2}\right\}}{\left(1-s^{2}\right)^{2}} \psi(s)=0
$$

By comparing Eq. (7) and Eq. (33), one can determine the following

$$
\tilde{\tau}(s)=-2 s, \quad \sigma(s)=1-s^{2}, \quad \widetilde{\sigma}(s)=1(l+1)\left(1-s^{2}\right)-m^{2}
$$

Substituting these expressions in Eq. (34) into Eq. (15), one has

$$
\pi(s)= \pm \sqrt{[l(l+1)-k] s^{2}+k+m^{2}-l(l+1)}
$$

Following the similar procedures in the radial equation, one can obtain

$$
\begin{gathered}
\mathrm{k}=-\mathrm{m}^{2}+\mathrm{l}(\mathrm{l}+1) \\
\pi(\mathrm{s})=-\mathrm{ms} \\
\tau(\mathrm{s})=-2(1+\mathrm{m}) \mathrm{s} \\
\lambda=-\mathrm{m}^{2}+\mathrm{l}(\mathrm{l}+1)-\mathrm{m} \\
\lambda_{\mathrm{n}}=2 \mathrm{n}+2 \mathrm{mn}+\mathrm{n}(\mathrm{n}-1) \\
\mathrm{n}=\mathrm{l}-\mathrm{m} \\
\phi(\mathrm{s})=\left(1-\mathrm{s}^{2}\right)^{\frac{\mathrm{m}}{2}}
\end{gathered}
$$




$$
\begin{gathered}
\rho(s)=\left(1-s^{2}\right)^{m} \\
\psi_{l, m}(s)=N_{l, m}\left[\left(1-s^{2}\right)^{\frac{m}{2}}\right]\left[\frac{1}{(1-m) !}\left(1-s^{2}\right)^{-m} \frac{d^{l-m}}{d s^{1-m}}\left\{\left(1-s^{2}\right)^{l}\right\}\right]
\end{gathered}
$$

where $N_{l, m}$ is the normalization constant.

\section{The Solution to Azimuthal Equation}

To solve Eq. (6), the boundary condition is set to fulfill

$$
\Phi_{\mathrm{m}}(\phi)=\Phi_{\mathrm{m}}(\phi+2 \pi)
$$

Therefore, the azimuthal equation has a solution as follows

$$
\Phi_{\mathrm{m}}(\phi)=\frac{1}{\sqrt{2 \pi}} \exp ( \pm \operatorname{im} \phi)
$$

where $\mathrm{i}$ is the imaginary number.

Based on these analytical results above, the wave function can be obtained for each quantum number of a single electron. By using Eq. (31) and Eq. (32), it is obtained the radial wave function as shown in Table 1. The angular and the azimuth terms can be obtained by using Eq. (44) and Eq. (46), as presented in Table 2. These obtained results are similar to the wave functions presented in quantum physics textbooks. It shows that the wave function and the energy eigenvalue of a single electron can be derived by using the NU method. This NU method provides an alternative way to solve the nonrelativistic Schrodinger equation analytically where this equation has second-order derivative. This

\begin{tabular}{|c|c|c|c|c|}
\hline $\mathrm{n}_{\mathrm{p}}$ & $\mathrm{n}$ & 1 & $\psi_{\mathrm{n}, \mathrm{l}}(\mathrm{r})$ & $\mathrm{R}_{\mathrm{n}_{\mathrm{p}, \mathrm{l}}}(\mathrm{r})$ \\
\hline 1 & 0 & 0 & $\Psi_{0,0}(r)$ & $\mathrm{R}_{1,0}=2\left(\frac{\mathrm{Z}}{\mathrm{a}_{0}}\right)^{\frac{3}{2}} \exp \left[-\frac{\mathrm{Zr}}{\mathrm{a}_{0}}\right]$ \\
\hline 2 & 1 & 0 & $\psi_{1,0}(r)$ & $\mathrm{R}_{2,0}=2\left(\frac{\mathrm{Z}}{2 \mathrm{a}_{0}}\right)^{\frac{3}{2}}\left(1-\frac{\mathrm{Zr}}{2 \mathrm{a}_{0}}\right) \exp \left[-\frac{\mathrm{Zr}}{2 \mathrm{a}_{0}}\right]$ \\
\hline 2 & 1 & 1 & $\psi_{0,1}(r)$ & $\mathrm{R}_{2,1}=\frac{1}{\sqrt{3}}\left(\frac{\mathrm{Z}}{2 \mathrm{a}_{0}}\right)^{\frac{3}{2}}\left(\frac{\mathrm{Zr}}{\mathrm{a}_{0}}\right) \exp \left[-\frac{\mathrm{Zr}}{2 \mathrm{a}_{0}}\right]$ \\
\hline 3 & 2 & 0 & $\psi_{2,0}(r)$ & $\mathrm{R}_{3,0}=2\left(\frac{\mathrm{Z}}{3 \mathrm{a}_{0}}\right)^{\frac{3}{2}}\left(1-\frac{2 \mathrm{Zr}}{3 \mathrm{a}_{0}}+\frac{2 \mathrm{Z}^{2} \mathrm{r}^{2}}{27 \mathrm{a}_{0}^{2}}\right) \exp \left[-\frac{\mathrm{Zr}}{3 \mathrm{a}_{0}}\right]$ \\
\hline 3 & 1 & 1 & $\psi_{1,1}(r)$ & $\mathrm{R}_{3,1}=\frac{4 \sqrt{2}}{3}\left(\frac{\mathrm{Z}}{3 \mathrm{a}_{0}}\right)^{\frac{3}{2}}\left(\frac{\mathrm{Zr}}{\mathrm{a}_{0}}-\frac{\mathrm{Z}^{2} \mathrm{r}^{2}}{6 \mathrm{a}_{0}^{2}}\right) \exp \left[-\frac{\mathrm{Zr}}{3 \mathrm{a}_{0}}\right]$ \\
\hline
\end{tabular}
method can be used not only for the Schrodinger equation, but also for every equation that has secondorder derivative.

Table 1. The radial wave function of a single electron. 
Table 2. The angular and the azimuth wave function for a single electron.

\begin{tabular}{cccc}
\hline $\mathrm{l}$ & $\mathrm{m}$ & $\Theta_{\mathrm{l}, \mathrm{m}}(\theta)$ & $\Phi_{\mathrm{m}}(\phi)$ \\
\hline 0 & 0 & $\frac{1}{\sqrt{2}}$ & $\frac{1}{\sqrt{2 \pi}}$ \\
1 & 0 & $\frac{\sqrt{3}}{\sqrt{2}} \cos \theta$ & $\frac{1}{\sqrt{2 \pi}}$ \\
1 & 1 & $\frac{\sqrt{3}}{2} \sin \theta$ & $\frac{1}{\sqrt{2 \pi}} \exp ( \pm \mathrm{i} \phi)$ \\
\hline
\end{tabular}

\section{CONCLUSION}

In this theoretical study, the Schrodinger equation has been solved analytically by using the NU method. The expressions of the wave function and the energy eigenvalue of a single electron have been also derived. The obtained expressions are in good agreement with the results lectured in the quantum physics textbooks. These results have proven that the NU method can provide an alternative way to solve the Schrodinger equation applied at the atomic level. This study can be extended to solve a system with many electrons with special treatment.

\section{REFERENCES}

Agboola, D. (2011). Schrodinger equation with Hulthen potential plus ring-shaped potential. Communications in Theoretical Physics, 55, 972-976.

Antia, A.D., Eze, C.C., \& Akpabio, L.E. (2018). Solutions of the Schrodinger equation with the harmonic oscillator potential (HOP) in cylindrical basis. Physics \& Astronomy International Journal, 2(3), 187-191.

Bayrak, O. \& Aciksoz, E. (2015). Corrected analytical solution of the generalized Woods-Saxon potential for arbitrary $l$ states. Physica Scripta, 90, 015302

Edet, C.O., Okorie, U.S., Ngiangia, A.T., \& Ikot, A.N. (2020). Bound state solutions of the Schrodinger equation for the modified Kratzer potential plus screened Coulomb potential. Indian Journal of Physics, 94, 425-433.

Eisberg, R. (1985). Quantum Mechanics of Atoms, Molecules, Solids, Nuclei, and Particles (2nd ed.). John Wiley and Sons.

Gasiorowicz, S. (2000). Quantum Physics (2nd ed.). John Wiley and Sons.

Griffith, D. (1995). Introduction to Quantum Mechanics. Prentice Hall.

Ikot, A.N., \& Akpan, I.O. (2012). Bound state solutions of the Schrodinger equations for a more general Woods-Saxon potential with arbitrary l-state. Chinese Physics Letters, 29, 09302.

Meyur, S. \& Debnath, S. (2009). Solution of the Schrodinger equation with Hulthen plus ManningRosen potential. Latin-American Journal of Physics Education, 3(2), 300-306.

Nikiforov, A.F. \& Uvarov, V.B. (1988). Special functions of mathematical physics. Birkhauser.

Okon, I.B., Popoola, O., \& Isonguyo, C.N. (2017). Approximation solutions of Schrodinger equation with some diatomic molecular interactions using Nikiforov-Uvarov method. Advances in High Energy Physics, 2017, 1-23. 
Pahlavani, M.R. \& Alavi, S.A. (2012). Solutions of Woods-Saxon potential with spin-orbit and centrifugal terms through Nikiforov-Uvarov method. Communications in Theoretical Physics, 58 , 739-743.

Sever, R., Tezcan, C., Aktas, M., \& Yesiltas, O. (2008). Exact solution of Schrodinger equation for pseudoharmonic potential. Journal of Mathematical Chemistry, 43(2), 845-851.

Zettili, N. (2009). Quantum Mechanics: concepts and applications 2nd Ed. Wiley

\section{Appendix 1}

\section{Radial part}

$$
\begin{aligned}
& R_{2,0}(r)=\psi_{2-0-1,0}(r)=\psi_{1,0}(r) \\
& \Psi_{1,0}(r)=N_{1,0}\left\{s^{0} e^{-\frac{Z r}{(1+0+1) a_{0}}}\right\}\left\{\frac{1}{1 !} e^{\frac{2 Z r}{(1+0+1) a_{0}}} r^{-(2 \cdot 0+1)} \frac{d^{1}}{d r^{1}}\left\{r^{1+2 \cdot 0+1} e^{-\frac{2 Z r}{(1+0+1) a_{0}}}\right\}\right\} \\
& \psi_{1,0}(r)=N_{1,0}\left\{e^{-\frac{Z r}{2 a_{0}}}\right\}\left\{e^{\frac{2 Z r}{2 a_{0}}} \frac{1}{r} \frac{d}{d r}\left[r^{2} e^{-\frac{2 Z r}{2 a_{0}}}\right]\right\} \\
& \psi_{1,0}(r)=N_{1,0}\left\{\frac{1}{r} e^{\frac{Z r}{2 a_{0}}}\right\}\left\{\frac{d}{d r}\left[r^{2} e^{-\frac{z r}{a_{0}}}\right]\right\} \\
& \Psi_{1,0}(r)=N_{1,0}\left\{\frac{1}{r} e^{\frac{Z r}{2 a_{0}}}\right\}\left\{e^{-\frac{Z r}{a_{0}}} \frac{d}{d r}\left[r^{2}\right]+r^{2} \frac{d}{d r}\left[e^{-\frac{z r}{a_{0}}}\right]\right\} \\
& \Psi_{1,0}(r)=N_{1,0}\left\{\frac{1}{r} e^{\frac{Z r}{2 a_{0}}}\right\}\left\{2 r e^{-\frac{Z r}{a_{0}}}-\frac{Z}{a_{0}} r^{2} e^{-\frac{z r}{a_{0}}}\right\} \\
& \psi_{1,0}(r)=N_{1,0} e^{\frac{Z r}{2 a_{0}}} e^{-\frac{Z r}{a_{0}}}\left\{2-\frac{Z r}{a_{0}}\right\} \\
& \psi_{1,0}(r)=N_{1,0} 2 e^{-\frac{Z r}{2 a_{0}}}\left\{1-\frac{Z r}{2 a_{0}}\right\} \\
& \int\left\{\mathrm{N}_{1,0} 2 \mathrm{e}^{-\frac{\mathrm{Zr}}{2 \mathrm{a}_{0}}}\left\{1-\frac{\mathrm{Zr}}{2 \mathrm{a}_{0}}\right\}\right\}^{2} \mathrm{r}^{2} \mathrm{dr}=1 \\
& \mathrm{~N}_{1,0}^{2} 4 \int\left\{\mathrm{e}^{-\frac{\mathrm{Zr}}{\mathrm{a}_{0}}}\left\{\mathrm{r}^{2}-\frac{2 \mathrm{Zr}^{3}}{2 \mathrm{a}_{0}}+\frac{\mathrm{Z}^{2} \mathrm{r}^{4}}{4 \mathrm{a}_{0}^{2}}\right\}\right\} \mathrm{dr}=1 \\
& N_{1,0}^{2} 4\left[\int r^{2} e^{-\frac{Z r}{a_{0}}} d r-\frac{Z}{a_{0}} \int r^{3} e^{-\frac{Z r}{a_{0}}} d r+\frac{Z^{2}}{4 a_{0}^{2}} \int r^{4} e^{-\frac{Z r}{a_{0}}} d r\right]=1 \\
& \mathrm{~N}_{1,0}^{2} 4\left[\frac{2 !}{\left(\frac{\mathrm{Z}}{\mathrm{a}_{0}}\right)^{3}}-\frac{\mathrm{Z}}{\mathrm{a}_{0}} \frac{3 !}{\left(\frac{\mathrm{Z}}{\mathrm{a}_{0}}\right)^{4}}+\frac{\mathrm{Z}^{2}}{4 \mathrm{a}_{0}^{2}} \frac{4 !}{\left(\frac{\mathrm{Z}}{\mathrm{a}_{0}}\right)^{5}}\right]=1 \\
& \mathrm{~N}_{1,0}^{2} 4\left[\frac{2}{\left(\frac{\mathrm{Z}}{\mathrm{a}_{0}}\right)^{3}}-\frac{6}{\left(\frac{\mathrm{Z}}{\mathrm{a}_{0}}\right)^{3}}+\frac{1}{4} \frac{24}{\left(\frac{\mathrm{Z}}{\mathrm{a}_{0}}\right)^{3}}\right]=1 \\
& \mathrm{~N}_{1,0}^{2} 4[2-6+6]=\left(\frac{\mathrm{Z}}{\mathrm{a}_{0}}\right)^{3} \\
& \mathrm{~N}_{1,0}^{2}=\frac{1}{8}\left(\frac{\mathrm{Z}}{\mathrm{a}_{0}}\right)^{3} \\
& \mathrm{~N}_{1,0}=\sqrt{\left(\frac{\mathrm{Z}}{2 \mathrm{a}_{0}}\right)^{3}}
\end{aligned}
$$




$$
\begin{aligned}
& \Psi_{1,0}(\mathrm{r})=\mathrm{N}_{1,0} 2 \mathrm{e}^{-\frac{\mathrm{Zr}}{2 \mathrm{a}_{0}}}\left\{1-\frac{\mathrm{Zr}}{2 \mathrm{a}_{0}}\right\} \\
& \Psi_{1,0}(\mathrm{r})=2\left(\frac{\mathrm{Z}}{2 \mathrm{a}_{0}}\right)^{\frac{3}{2}}\left\{1-\frac{\mathrm{Zr}}{2 \mathrm{a}_{0}}\right\} \mathrm{e}^{-\frac{\mathrm{Zr}}{2 \mathrm{a}_{0}}}
\end{aligned}
$$

\section{Angular part}

$$
\begin{aligned}
& \psi_{\mathrm{l}, \mathrm{m}}(\cos \theta)=\mathrm{N}_{\mathrm{l}, \mathrm{m}}\left[\left(1-\cos ^{2} \theta\right)^{\frac{\mathrm{m}}{2}}\right] \\
& \times\left[\frac{1}{(1-m) !}\left(1-\cos ^{2} \theta\right)^{-m} \frac{d^{1-m}}{d(\cos \theta)^{1-m}}\left\{\left(1-\cos ^{2} \theta\right)^{1}\right\}\right] \\
& \psi_{1,1}(\cos \theta)=N_{1,1}\left[\left(1-\cos ^{2} \theta\right)^{\frac{1}{2}}\right]\left[\frac{1}{(1-1) !}\left(1-\cos ^{2} \theta\right)^{-1} \frac{d^{1-1}}{d(\cos \theta)^{1-1}}\left\{\left(1-\cos ^{2} \theta\right)^{1}\right\}\right] \\
& \psi_{1,1}(\cos \theta)=N_{1,1}\left[\left(1-\cos ^{2} \theta\right)^{\frac{1}{2}}\right] \\
& \psi_{1,1}(\cos \theta)=N_{1,1}\left[\left(\sin ^{2} \theta\right)^{\frac{1}{2}}\right] \\
& \psi_{1,1}(\cos \theta)=\mathrm{N}_{1,1}[\sin \theta] \\
& \int_{0}^{\pi}\left\{\mathrm{N}_{1,1}[\sin \theta]\right\}^{2} \sin \theta d \theta=1 \\
& \mathrm{~N}_{1,1}^{2} \int_{0}^{\pi} \sin ^{2} \theta \sin \theta d \theta=1 \\
& \mathrm{~N}_{1,1}^{2}\left[\frac{1}{12} \cos 3 \theta-\frac{3}{4} \cos \theta\right]_{0}^{\pi}=1 \\
& \mathrm{~N}_{1,1}^{2}\left[\frac{4}{3}\right]=1 \\
& \mathrm{~N}_{1,1}^{2}=\frac{3}{4} \\
& \mathrm{~N}_{1,1}=\frac{\sqrt{3}}{2} \\
& \psi_{1,1}(\cos \theta)=\mathrm{N}_{1,1}[\sin \theta]=\frac{\sqrt{3}}{2} \sin \theta
\end{aligned}
$$

